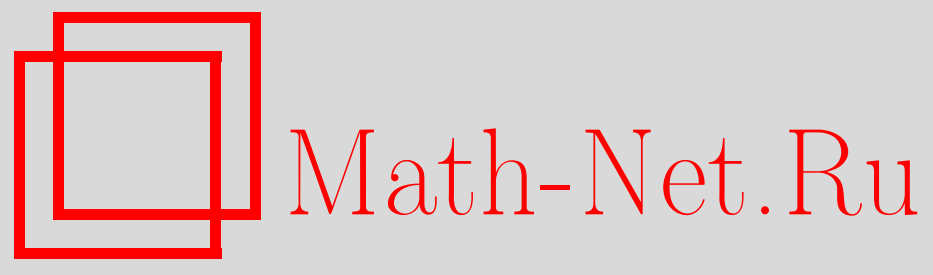

N. Guillotin-Plantard, A. Le Ny, Transient Random Walks on 2D-Oriented Lattices, Теория вероятн. и ее примен., 2007, том 52, выпуск 4, 815-826

DOI: https://doi.org/10.4213/tvp1651

Использование Общероссийского математического портала MathNet.Ru подразумевает, что вы прочитали и согласны с пользовательским соглашением

http://www . mathnet.ru/rus/agreement

Параметры загрузки:

IP : 54.172 .240 .79

26 апреля 2023 г., 11:35:14

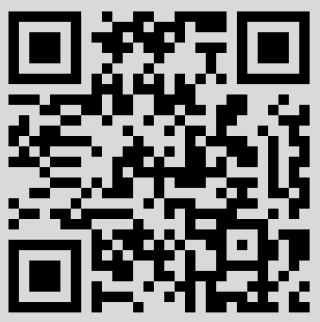


(C) 2007 r. GUILLOTIN-PLANTARD N.*, LE NYA.**

\title{
TRANSIENT RANDOM WALKS ON 2D-ORIENTED LATTICES ${ }^{1)}$
}

\begin{abstract}
Изучается асимптотическое поведение простого случайного блуждания на ориентированных версиях решетки $\mathbf{Z}^{2}$. Рассматриваемые решетки не ориентированы по вертикальной оси, ориентация же по поризонтальной оси случайна с распределениями, порождаемыми динамической системой. Найдено условие на гладкость порождающей функции, являющееся достаточным для транзиентности простого случайного блуждания на почти всех так ориентированных решетках. В качестве иллюстрации предлагается широкий класс примеров неоднородных или коррелированных распределений ориентаций. Для эргодических динамических систем установлен усиленный закон больших чисел и, в частном случае независимых и одинаково распределенных ориентаций, решена одна открытая проблема, а именно, в пространстве $\mathscr{D}\left(\left[0, \infty\left[, \mathbf{R}^{2}\right)\right.\right.$ функций, непрерывных справа и имеющих предел слева, доказана функциональная предельная теорема с нестандартным нормированием.
\end{abstract}

Ключевые слова и фразы: случайные блуждания, случайные среды, случайные сценарии, ориентированные графы, динамические системы, рекуррентность, транзиентность, предельные теоремы.

1. Introduction. The use of random walks as a tool in mathematical physics is now well established and they have been, for example, widely used in classical statistical mechanics to study critical phenomena [4]. Analogous methods in quantum statistical mechanics require the study of random walks on oriented lattices, due to the intrinsic noncommutative character of the (quantum) world [3], [13]. Although random walks in random and nonrandom environments have been intensively studied for many years, only a few results on random walks on oriented lattices are known. The recurrence or transience properties of simple random walks on oriented versions of $\mathbf{Z}^{2}$ are studied in [2] when the horizontal lines are unidirectional towards a random or deterministic direction. An interesting feature of this model is that, depending on the orientation, the walk could be either recurrent or transient. In a particular deterministic case, alternatively oriented horizontally rightwards and leftwards, the recurrence of the simple random walk is proved, whereas the transience naturally arises when the orientations are all identical in infinite regions. More surprisingly, it is also proved that the recurrent character of the simple random walk on $\mathbf{Z}^{2}$ is lost when the orientations are i.i.d. with zero mean.

In this paper, we study more general models and focus on spatially inhomogeneous or dependent distributions of the orientations. We introduce lattices for which the distribution of the orientation is generated by a dynamical system and prove that the transience of the simple random walk still holds under smoothness conditions on this generation. We detail examples and counterexamples for various standard dynamical systems. For ergodic dynamical systems, we also prove a strong law of large numbers and, in the case of i.i.d. orientations, a functional limit theorem with an unconventional normalization due to the random character of the environment of the walk, solving an open question of [2].

The model and our results are stated in Section 2, Section 3 is devoted to the proofs, while illustrative examples of such dynamical orientations are given in Section 4 .

* Université Claude Bernard-Lyon I, Institut Camille Jordan, bâtiment Braconnier, 43, avenue du 11 novembre 1918, 69622 Villeurbanne Cedex, France; e-mail: nadine.guillotin@univ-lyon1.fr

** Université de Paris-Sud, Laboratoire de mathématiques, bât. 425, 91405 Orsay Cedex, France; e-mail: arnaud.leny@math.u-psud.fr

1) The first author was partially supported by the RDSES program of the ESF. 


\section{Model and results.}

2.1. Dynamically oriented lattices. Let $S=(E, \mathscr{A}, \mu, T)$ be a dynamical system, where $(E, \mathscr{A}, \mu)$ is a probability space and $T$ is an invertible transformation of $E$ preserving the measure $\mu$. This system is used to introduce inhomogeneity or dependencies in the distribution of the random orientations, together with a function $f$ from $E$ to $[0,1]$, which satisfies $\int_{E} f d \mu=\frac{1}{2}$ to avoid trivialities. By orientations, we mean a random field $\epsilon=$ $\left(\epsilon_{y}\right)_{y \in \mathbf{Z}} \in\{-1,+1\}^{\mathbf{Z}}$, i.e., a family $\epsilon$ of $\{-1,+1\}$-valued random variables $\epsilon_{y}, y \in \mathbf{Z}$, and we distinguish two different approaches to introduce its distribution.

2.1.1. Quenched case. It describes orientations whose distributions are spatially inhomogeneous. For $x \in E$ the quenched law $\mathbf{P}_{T}^{(x)}$ is the product probability measure on $\{-1,+1\}^{\mathbf{Z}}$, equipped with the product $\sigma$-algebra $\mathscr{F}=\mathscr{P}(\{-1,+1\})^{\otimes \mathbf{Z}}$, whose marginals can be given by

$$
\mathbf{P}_{T}^{(x)}\left[\epsilon_{y}=+1\right]=f\left(T^{y} x\right) .
$$

For simplicity, we have used the same notation for the quenched law and its marginals, which should be written $\mathbf{P}_{T, y}^{(x)}$ with $\mathbf{P}_{T}^{(x)}=\bigotimes_{y} \mathbf{P}_{T, y}^{(x)}$. This quenched case is an extension of the i.i.d. case, with independent but not necessarily identically distributed random variables. These random variables can be viewed as the increments of a dynamic random walk [5], [6].

2.1.2. Annealed case. We average on $x \in E$ : the distribution of $\epsilon$ is now $\mathbf{P}_{\mu}$ defined for all $A \in \mathscr{F}$ by

$$
\mathbf{P}_{\mu}[\epsilon \in A]=\int_{E} \mathbf{P}_{T}^{(x)}[\epsilon \in A] d \mu(x) .
$$

The marginals are thus given for all $y \in \mathbf{Z}$ by

$$
\mathbf{P}_{\mu}\left[\epsilon_{y}=+1\right]=\int_{E} f\left(T^{y} x\right) d \mu(x)=\int_{E} f(x) d \mu(x)=\frac{1}{2}
$$

and the hypothesis $\int_{E} f d \mu=\frac{1}{2}$ has been taken to get $\mathbf{E}_{\mu}\left[\epsilon_{y}\right]=0$. The $T$-invariance of $\mu$ implies the translation-invariance of $\mathbf{P}_{\mu}$ but this latter is not a product measure in general: The correlations of the dynamical system for the function $f$, defined for all $y \in \mathbf{Z}$ by

$$
\begin{aligned}
C_{\mu}^{f}(y) & :=\int_{E} f(x) \cdot f \circ T^{y}(x) d \mu(x)-\int_{E} f(x) d \mu(x) \cdot \int_{E} f \circ T^{y}(x) d \mu(x) \\
& =\int_{E} f(x) f\left(T^{y}(x)\right) d \mu(x)-\frac{1}{4}
\end{aligned}
$$

are indeed related, as a short computation shows with the covariance of the $\epsilon$ 's:

$$
\forall y \in \mathbf{Z} \quad \operatorname{Cov}_{\mu}\left(\epsilon_{0}, \epsilon_{y}\right)=4 C_{\mu}^{f}(y) .
$$

One can thus construct dependent variables whose dependence is directly related to the correlations of the dynamical system. This annealed case leads in Section 4 to another extension of the i.i.d. case, where independence is dropped but translation-invariance is kept.

2.1.3. Lattices. We use these orientations to build dynamically oriented lattices. They are oriented versions of $\mathbf{Z}^{2}$ : the vertical lines are not oriented and the horizontal ones are unidirectional, the orientation at a level $y \in \mathbf{Z}$ being given by the random variable $\epsilon_{y}$ (say right if the value is +1 and left if it is -1 ). More formally we give the following definition.

D e f i n i t i o n 1 (dynamically oriented lattices). Let $\epsilon=\left(\epsilon_{y}\right)_{y \in Z}$ be a sequence of orientations defined as previously. The dynamically oriented lattice $\mathbb{L}^{\epsilon}=\left(\mathbb{V}, \mathbb{A}^{\epsilon}\right)$ is the (random) directed graph with (deterministic) vertex set $\mathbb{V}=\mathbf{Z}^{2}$ and (random) edge set $\mathbb{A}^{\epsilon}$ defined by the condition that for $u=\left(u_{1}, u_{2}\right), v=\left(v_{1}, v_{2}\right) \in \mathbf{Z}^{2}$, the edge $(u, v)$ belongs to $\mathbb{A}^{\epsilon}$ if and only if $v_{1}=u_{1}$ and $v_{2}=u_{2} \pm 1$, or $v_{2}=u_{2}$ and $v_{1}=u_{1}+\epsilon_{u_{2}}$. 
2.2. Simple random walk on $\mathbb{L}^{\epsilon}$. We consider the simple random walk $M=\left(M_{n}\right)_{n \in \mathbf{N}}$ on $\mathbb{L}^{\epsilon}$. For every realization of $\epsilon$, it is a $\mathbf{Z}^{2}$-valued Markov chain defined on a probability space $(\Omega, \mathscr{B}, \mathbf{P})$, whose $(\epsilon$-dependent) transition probabilities are defined for all $(u, v) \in \mathbb{V} \times \mathbb{V}$ by

$$
\mathbf{P}\left[M_{n+1}=v \mid M_{n}=u\right]= \begin{cases}\frac{1}{3} & \text { if }(u, v) \in \mathbb{A}^{\epsilon} \\ 0 & \text { otherwise. }\end{cases}
$$

Its transience is proved in [2] for almost every orientation $\epsilon$ when the $\epsilon_{y}$ 's are i.i.d. and we generalize it in this dynamical context when the orientations are either annealed or quenched.

Theorem 1. Assume that

$$
\int_{E} \frac{1}{\sqrt{f(1-f)}} d \mu<\infty
$$

Then

1) in the annealed case, for $\mathbf{P}_{\mu}$-a.e. orientation $\epsilon$, the simple random walk on dynamically oriented lattice $\mathbb{L}^{\epsilon}$ is transient;

2) in the quenched case, for $\mu$-a.e. $x \in E$, for $\mathbf{P}_{T}^{(x)}$-a.e. realization of the orientation $\epsilon$, the simple random walk on the dynamically oriented lattice $\mathbb{L}^{\epsilon}$ is transient.

$\mathrm{R}$ e m a r k s. 1. Noninvertible transformations $T$ of the space $E$ can also be considered and in this case it is straightforward to extend the conclusions of Theorem 1 if the distribution of the orientations $\left(\epsilon_{y}\right)_{y \in \mathbf{Z}}$ have marginals defined by $\mathbf{P}_{T}^{(x)}\left[\epsilon_{y}=+1\right]=f\left(T^{|y|} x\right)$. The measure $\mathbf{P}_{\mu}$ is not stationary anymore in the annealed case (see the example of Manneville-Pomeau maps of the interval in Section 4).

2. In Section 4 we exhibit dynamical systems for which Theorem 1 applies (Bernoulli or Markov shifts, Manneville-Pomeau maps), but also counterexamples from a family of dynamical systems (irrational and rational rotations on the torus). The latter case provides instructive examples: when the function $f$ satisfies (2.3), the ergodicity (or nonergodicity) of the dynamical system is not required, whereas when $f$ does not fulfil (2.3), the properties of the underlying dynamical system can play a role, e.g., in the nonergodic case when, according to the rational angle we choose, the simple random walk on the corresponding oriented lattice can be transient or recurrent.

2.3. Limit theorems in the ergodic case. Let us assume that the dynamical system $S=(E, \mathscr{A}, \mu, T)$ defined in Section 2.1 is ergodic. We denote by $\widetilde{\mathbf{P}}_{\mu}$ the measure $\int \mathbf{P}[\cdot] d \mathbf{P}_{\mu}$.

Theorem 2 (strong law of large numbers). The random walk on the lattice $\mathbb{L}^{\epsilon}$ has $\widetilde{\mathbf{P}}_{\mu}$-almost surely zero speed, i.e.,

$$
\lim _{n \rightarrow+\infty} \frac{M_{n}}{n}=(0,0) \quad \widetilde{\mathbf{P}}_{\mu} \text {-a.s. }
$$

2.3.1. Functional limit theorem for i.i.d. orientations. In this paper we also answer an open question of [2] and obtain a functional limit theorem with a suitable normalization. We establish that the study of the simple random walk on $\mathbb{L}^{\epsilon}$ is closely related to a simple random walk in a random scenery defined for every $n \geqslant 1$ by

$$
Z_{n}=\sum_{k=0}^{n} \epsilon_{Y_{k}},
$$

where $\left(Y_{k}\right)_{k \geqslant 0}$ is the simple random walk on $\mathbf{Z}$ starting from 0 . Consider a standard Brownian motion $\left(B_{t}\right)_{t \geqslant 0}$, denote by $\left(L_{t}(x)\right)_{t \geqslant 0}$ its corresponding local time at $x \in \mathbf{R}$, and introduce a pair of independent Brownian motions $\left(Z_{+}(x), Z_{-}(x)\right), x \geqslant 0$, defined on the same probability space as $\left(B_{t}\right)_{t \geqslant 0}$ and independent of it. The following process is well defined for all $t \geqslant 0$ :

$$
\Delta_{t}=\int_{0}^{\infty} L_{t}(x) d Z_{+}(x)+\int_{0}^{\infty} L_{t}(-x) d Z_{-}(x) .
$$


It has been proven in [12] that this process has a self-similar continuous version of index $\frac{3}{4}$, with stationary increments. The symbol $\stackrel{\mathscr{D}}{\longrightarrow}$ denotes convergence in the space of càdlàg functions $\mathscr{D}([0, \infty), \mathbf{R})$ endowed with the Skorokhod topology.

Theorem 3 ([12]). We have

$$
\left(\frac{1}{n^{3 / 4}} Z_{[n t]}\right)_{t \geqslant 0} \stackrel{\mathscr{D}}{\longrightarrow}\left(\Delta_{t}\right)_{t \geqslant 0}
$$

We introduce a real constant $m=\frac{1}{2}$, defined later as the mean of some geometric random variables related to the behavior of the walk in the horizontal direction ${ }^{1)}$. Using Theorem 3 we shall prove the following result.

Theorem 4 (functional limit theorem). We have

$$
\left(\frac{1}{n^{3 / 4}} M_{[n t]}\right)_{t \geqslant 0} \stackrel{\mathscr{D}}{\rightarrow} \frac{m}{(1+m)^{3 / 4}}\left(\Delta_{t}, 0\right)_{t \geqslant 0}
$$

$\mathrm{R}$ e $\mathrm{m}$ a $\mathrm{r} \mathrm{k}$. It is not surprising that the vertical component is negligible towards $n^{3 / 4}$ because its fluctuations are of order $\sqrt{n}$. We suspect that we have in fact

$$
\left(\frac{1}{n^{3 / 4}} M_{[n t]}^{(1)}, \frac{1}{n^{1 / 2}} M_{[n t]}^{(2)}\right)_{t \geqslant 0} \stackrel{\mathscr{D}}{\longrightarrow}\left(\frac{m}{(1+m)^{3 / 4}} \Delta_{t}, B_{t}\right)_{t \geqslant 0}
$$

but this is not straightforward because the horizontal $\left(M^{(1)}\right)$ and vertical $\left(M^{(2)}\right)$ components are not independent. We believe that $\left(B_{t}\right)_{t \geqslant 0}$ and $\left(\Delta_{t}\right)_{t \geqslant 0}$ are independent but this also has to be proved.

\section{Proofs.}

3.1. Vertical and horizontal embeddings of the simple random walk. The simple random walk $M$ defined on $(\Omega, \mathscr{B}, \mathbf{P})$ can be decomposed into vertical and horizontal embeddings by projection onto the corresponding axis. The vertical one is a simple random walk $Y=\left(Y_{n}\right)_{n \in \mathbf{N}}$ on $\mathrm{Z}$ and we define for all $n \in \mathbf{N}$ its local time at the level $y \in \mathbf{Z}$ by

$$
\eta_{n}(y)=\sum_{k=0}^{n} 1\left\{Y_{k}=y\right\}
$$

The horizontal embedding is a random walk with $\mathrm{N}$-valued geometric jumps: a doubly

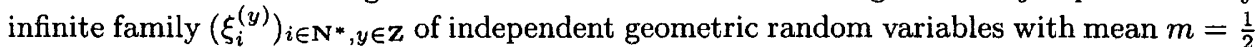
is given and one defines the embedded horizontal random walk $X=\left(X_{n}\right)_{n \in \mathrm{N}}$ by $X_{0}=0$ and for $n \geqslant 1$,

$$
X_{n}=\sum_{y \in \mathbf{Z}} \epsilon_{y} \sum_{i=1}^{\eta_{n-1}(y)} \xi_{i}^{(y)}
$$

with the convention that the last sum is zero when $\eta_{n-1}(y)=0$. Of course, the walk $M_{n}$ does not coincide with $\left(X_{n}, Y_{n}\right)$ but these objects are closely related: define for all $n \in \mathbf{N}$

$$
T_{n}=n+\sum_{y \in \mathbf{Z}} \sum_{i=1}^{\eta_{n-1}(y)} \xi_{i}^{(y)}
$$

to be the instant just after the random walk $M$ has performed its $n$-th vertical move. A direct and useful consequence of this decomposition is the following result [2].

Lemma 1. 1. $M_{T_{n}}=\left(X_{n}, Y_{n}\right)$, for all $n \in \mathbf{N}$.

2. For a given orientation $\epsilon$, the transience of $\left(M_{T_{n}}\right)_{n \in N}$ implies the transience of $\left(M_{n}\right)_{n \in N}$.

1) Our results are in fact valid for similar model, for which $m \neq \frac{1}{2}$, corresponding to nonsymmetric nearest neighbors random walks. Of course, the transience is not at all surprising in this case, but getting the limit theorems can be of interest. 
3.2. Proof of the transience of the simple random walk. The vertical walk $Y$, independent of $\epsilon$, is known to be recurrent with fluctuations of order $\sqrt{n}$. For any $i \in \mathbf{N}$, $\delta_{i}$ is a strictly positive real number and we write $d_{n, i}=n^{1 / 2+\delta_{i}}$ to introduce a partition of $\Omega$ into typical or untypical paths of $Y$ :

$$
A_{n}=\left\{\omega \in \Omega: \max _{0 \leqslant k \leqslant 2 n}\left|Y_{k}\right|<d_{n, 1}\right\} \cap\left\{\omega \in \Omega: \max _{y \in \mathbf{Z}} \eta_{2 n-1}(y)<d_{n, 2}\right\}
$$

and

$$
B_{n}=\left\{\omega \in A_{n}:\left|\sum_{y \in \mathbf{Z}} \epsilon_{y} \eta_{2 n-1}(y)\right|>d_{n, 3}\right\} \text {. }
$$

We first consider the joint measures $\widetilde{\mathbf{P}}_{\mu}=\int \mathbf{P}[\cdot] d \mathbf{P}_{\mu}$ (annealed case) or $\widetilde{\mathbf{P}}_{T}^{(x)}=$ $\int \mathbf{P}[\cdot] d \mathbf{P}_{T}^{(x)}$ (quenched case) and prove that

$$
\sum_{n \in \mathbb{N}} \tilde{\mathbf{P}}_{\mu}\left[X_{2 n}=0 ; Y_{2 n}=0\right]<\infty
$$

By definition,

$$
\sum_{n \in \mathbf{N}} \widetilde{\mathbf{P}}_{\mu}\left[X_{2 n}=0 ; Y_{2 n}=0\right]=\int_{E} \sum_{n} \mathbf{P}\left[\mathbf{P}_{T}^{(x)}\left[X_{2 n}=0 ; Y_{2 n}=0\right]\right] d \mu(x)
$$

and we first decompose $\widetilde{\mathbf{P}}_{T}^{(x)}\left[X_{2 n}=0 ; Y_{2 n}=0\right]$ into

$$
\begin{aligned}
\widetilde{\mathbf{P}}_{T}^{(x)} & {\left[X_{2 n}=0 ; Y_{2 n}=0 ; A_{n}^{c}\right]+\widetilde{\mathbf{P}}_{T}^{(x)}\left[X_{2 n}=0 ; Y_{2 n}=0 ; B_{n}\right] } \\
& +\widetilde{\mathbf{P}}_{T}^{(x)}\left[X_{2 n}=0 ; Y_{2 n}=0 ; A_{n} \backslash B_{n}\right] .
\end{aligned}
$$

Some results for the i.i.d. case stated in [2] still hold uniformly in $x$ and, in particular, using standard techniques, we can prove the following lemma.

Lemma 2. 1. For every $x \in E, \sum_{n \in \mathbf{N}} \widetilde{\mathbf{P}}_{T}^{(x)}\left[X_{2 n}=0 ; Y_{2 n}=0 ; A_{n}^{c}\right]<\infty$.

2. For every $x \in E, \sum_{n \in \mathbb{N}} \widetilde{\mathbf{P}}_{T}^{(x)}\left[X_{2 n}=0 ; Y_{2 n}=0 ; B_{n}\right]<\infty$.

Define the $\sigma$-algebras $\mathscr{F}=\sigma(Y)$ and $\mathscr{G}=\sigma(\epsilon)$ generated by the families of r.v.'s $Y$ and $\epsilon$, respectively. Then one has

$$
\begin{aligned}
p_{n}^{(x)}: & =\widetilde{\mathbf{P}}_{T}^{(x)}\left[X_{2 n}=0 ; Y_{2 n}=0 ; A_{n} \backslash B_{n}\right] \\
& =\mathbf{E}\left[\mathbf{1}\left\{Y_{2 n}=0\right\} \mathbf{E}\left[\mathbf{1}_{A_{n} \backslash B_{n}} \widetilde{\mathbf{P}}_{T}^{(x)}\left[X_{2 n}=0 \mid \mathscr{F} \vee \mathscr{G}\right] \mid \mathscr{F}\right]\right] .
\end{aligned}
$$

To prove Theorem 1, it remains to show that

$$
\int_{E}\left(\sum_{n \in \mathbf{N}} p_{n}^{(x)}\right) d \mu(x)<\infty
$$

Recall that for the simple random walk $Y$, there exists $C>0$ such that

$$
\mathbf{P}\left[Y_{2 n}=0\right] \sim C n^{-1 / 2}, \quad n \rightarrow+\infty,
$$

and we can prove as in [2] the following lemma.

Lemma 3. On the set $A_{n} \backslash B_{n}$, we have, uniformly in $x \in E$,

$$
\widetilde{\mathbf{P}}_{T}^{(x)}\left[X_{2 n}=0 \mid \mathscr{F} \vee \mathscr{G}\right]=O\left(\sqrt{\frac{\ln n}{n}}\right) .
$$

Hence, the transience of the simple random walk is a direct consequence of the following proposition. that

Proposition 1. It is possible to choose $\delta_{1}, \delta_{2}, \delta_{3}>0$ for which there exists $\delta>0$ such

$$
\int_{E} \widetilde{\mathbf{P}}_{T}^{(x)}\left[A_{n} \backslash B_{n} \mid \mathscr{F}\right] d \mu(x)=O\left(n^{-\delta}\right) .
$$


P r o of. We have to estimate, on the event $A_{n}$, the conditional probability

$$
\widetilde{\mathbf{P}}_{T}^{(x)}\left[\left|\sum_{y \in \mathbf{Z}} \zeta_{y}\right| \leqslant d_{n, 3} \mid \mathscr{F}\right]
$$

where $\zeta_{y}=\epsilon_{y} \eta_{2 n-1}(y), y \in \mathbf{Z}$. Let $G$ be a centered Gaussian random variable with variance $d_{n, 3}^{2}$ (conditionally on $\mathscr{F}$ ) independent of the random variables $\zeta_{y}$. Clearly,

$$
\widetilde{\mathbf{P}}_{T}^{(x)}\left[\sum_{y} \zeta_{y} \in\left[0, d_{n, 3}\right] \mid \mathscr{F}\right]=\frac{\widetilde{\mathbf{P}}_{T}^{(x)}\left[\sum_{y} \zeta_{y} \in\left[0, d_{n, 3}\right] ; 0 \leqslant G \leqslant d_{n, 3} \mid \mathscr{F}\right]}{\widetilde{\mathbf{P}}_{T}^{(x)}\left[0 \leqslant G \leqslant d_{n, 3} \mid \mathscr{F}\right]}
$$

where $\widetilde{\mathbf{P}}_{T}^{(x)}\left[0 \leqslant G \leqslant d_{n, 3} \mid \mathscr{F}\right]=c>0$ is independent of $n$. Since $G$ is independent of the random variables $\zeta_{y}$ and using the symmetry of the Gaussian distribution, we have

$\widetilde{\mathbf{P}}_{T}^{(x)}\left[\sum_{y} \zeta_{y} \in\left[0, d_{n, 3}\right] ; 0 \leqslant G \leqslant d_{n, 3} \mid \mathscr{F}\right]=\widetilde{\mathbf{P}}_{T}^{(x)}\left[\sum_{y} \zeta_{y} \in\left[0, d_{n, 3}\right] ;-d_{n, 3} \leqslant G \leqslant 0 \mid \mathscr{F}\right]$.

Consequently, we obtain

$$
\begin{array}{r}
\widetilde{\mathbf{P}}_{T}^{(x)}\left[\sum_{y} \zeta_{y} \in\left[0, d_{n, 3}\right] \mid \mathscr{F}\right] \leqslant \frac{1}{c} \widetilde{\mathbf{P}}_{T}^{(x)}\left[\left|\sum_{y} \zeta_{y}+G\right| \leqslant d_{n, 3} \mid \mathscr{F}\right], \\
\widetilde{\mathbf{P}}_{T}^{(x)}\left[\sum_{y} \zeta_{y} \in\left[-d_{n, 3}, 0\right] \mid \mathscr{F}\right] \leqslant \frac{1}{c} \tilde{\mathbf{P}}_{T}^{(x)}\left[\left|\sum_{y} \zeta_{y}+G\right| \leqslant d_{n, 3} \mid \mathscr{F}\right]
\end{array}
$$

and then, we have the following inequality:

$$
\widetilde{\mathbf{P}}_{T}^{(x)}\left[\left|\sum_{y} \zeta_{y}\right| \leqslant d_{n, 3} \mid \mathscr{F}\right] \leqslant \frac{2}{c} \widetilde{\mathbf{P}}_{T}^{(x)}\left[\left|\sum_{y} \zeta_{y}+G\right| \leqslant d_{n, 3} \mid \mathscr{F}\right] .
$$

From Plancherel's formula, we deduce that there exists a constant $C>0$ such that

$$
\tilde{\mathbf{P}}_{T}^{(x)}\left[\left|\sum_{y} \zeta_{y}+G\right| \leqslant d_{n, 3} \mid \mathscr{F}\right] \leqslant C d_{n, 3} I_{n}(x)
$$

where

$$
I_{n}(x)=\int_{-\pi}^{\pi} \mathbf{E}\left[\exp \left\{i t \sum_{y \in Z} \epsilon_{y} \eta_{2 n-1}(y)\right\} \mid \mathscr{F}\right] e^{-t^{2} d_{n, 3}^{2} / 2} d t .
$$

To use that for $t d_{n, 3}$ small enough, $e^{-t^{2} d_{n, 3}^{2} / 2}$ dominates the term under the expectation, we split the integral into two parts. For $b_{n}=n^{\delta_{2}} / d_{n, 3}$, we write $I_{n}(x)=I_{n}^{1}(x)+I_{n}^{2}(x)$ with

$$
\begin{aligned}
& I_{n}^{1}(x)=\int_{|t| \leqslant b_{n}} \mathbf{E}\left[\exp \left\{i t \sum_{y \in \mathbf{Z}} \epsilon_{y} \eta_{2 n-1}(y)\right\} \mid \mathscr{F}\right] e^{-t^{2} d_{n, 3}^{2} / 2} d t \\
& I_{n}^{2}(x)=\int_{|t|>b_{n}} \mathbf{E}\left[\exp \left\{i t \sum_{y \in \mathbf{Z}} \epsilon_{y} \eta_{2 n-1}(y)\right\} \mid \mathscr{F}\right] e^{-t^{2} d_{n, 3}^{2} / 2} d t .
\end{aligned}
$$

To control the integral $I_{n}^{2}(x)$, we write

$$
\left|I_{n}^{2}(x)\right| \leqslant C \int_{|t|>b_{n}} e^{-t^{2} d_{n, 3}^{2} / 2} d t=\frac{C}{d_{n, 3}} \int_{|s|>n^{\delta_{2}}} e^{-s^{2} / 2} d s \leqslant \frac{2 C}{d_{n, 3}} n^{-\delta_{2}} e^{-n^{2 \delta_{2} / 2}}
$$

and thus get uniformly in $x \in E$

$$
\left|I_{n}^{2}(x)\right|=O\left(e^{-n^{2 \delta_{2}} / 2}\right)
$$

Lemma 4. For $\delta_{3}>2 \delta_{2}$,

$$
\int_{E}\left|I_{n}^{1}(x)\right| d \mu(x)=O\left(n^{-3 / 4+\delta_{1} / 2}\right) .
$$


P r o o f. From the definition of the orientations $\left(\epsilon_{y}\right)_{y \in \mathbf{Z}}$, an explicit formula for the characteristic function $\phi_{\epsilon_{y}}^{(x)}$ of the random variable $\epsilon_{y}$ can be given, and we deduce that

$$
\left|\phi_{\epsilon_{y}}^{(x)} u\right|^{2}=\cos ^{2} u+\left(2 f\left(T^{y} x\right)-1\right)^{2} \sin ^{2} u=1-4 f\left(T^{y} x\right)\left(1-f\left(T^{y} x\right)\right) \sin ^{2} u
$$

and by independence of the $\epsilon$ 's we get

$$
\left|I_{n}^{1}(x)\right| \leqslant \int_{|t| \leqslant b_{n}}\left|\prod_{y} \phi_{\epsilon_{y}}^{(x)}\left(\eta_{2 n-1}(y) t\right)\right| d t .
$$

Denote $p_{n, y}=\eta_{2 n-1}(y) /(2 n), C_{n}=\left\{y: \eta_{2 n-1}(y) \neq 0\right\}$ and use Hölder's inequality to get

$$
\left|I_{n}^{1}(x)\right| \leqslant \prod_{y}\left[\left(\int_{|t| \leqslant b_{n}}\left|\phi_{\epsilon_{y}}^{(x)}\left(\eta_{2 n-1}(y) t\right)\right|^{1 / p_{n, y}} d t\right)^{p_{n, y}}\right] .
$$

Now, using the fact that we work on $A_{n}$, we choose $\delta_{3}>2 \delta_{2}$ so that $\lim _{n} b_{n} \eta_{2 n-1}(y)=0$ uniformly in $y$. Using inequalities $\sin x \geqslant 2 \pi^{-1} x$ for $x \in[0, \pi / 2]$ and $\exp (-x) \geqslant 1-x$, one has

$$
\begin{aligned}
\left|I_{n}^{1}(x)\right| & \leqslant \prod_{y \in C_{n}}\left(\frac{1}{\eta_{2 n-1}(y)} \int_{|v| \leqslant b_{n} \eta_{2 n-1}(y)}^{\exp }\left(-\frac{16}{p_{n, y} \pi^{2}} f\left(T^{y} x\right)\left(1-f\left(T^{y} x\right)\right) v^{2}\right) d v\right)^{p_{n, y}} \\
& \leqslant \prod_{y \in C_{n}}\left(\frac{c 1\left\{f\left(T^{y} x\right)\left(1-f\left(T^{y} x\right)\right)>0\right\}}{\sqrt{2 n \eta_{2 n-1}(y) f\left(T^{y} x\right)\left(1-f\left(T^{y} x\right)\right)}}\right)^{p_{n, y}} \quad\left(\text { with } c=\frac{\pi^{3 / 2}}{4}\right) \\
& =c \exp \left\{-\frac{1}{2} \sum_{y \in C_{n}} p_{n, y} \ln \left(2 n \eta_{2 n-1}(y)\right)\right\} \prod_{y \in C_{n}}\left(\frac{1\left\{f\left(T^{y} x\right)\left(1-f\left(T^{y} x\right)\right)>0\right\}}{\sqrt{f\left(T^{y} x\right)\left(1-f\left(T^{y} x\right)\right)}}\right)^{p_{n, y}} .
\end{aligned}
$$

The vector $\mathbf{p}=\left(p_{n, y}\right)_{y \in C_{n}}$ defines a probability measure on $C_{n}$ and we have

$$
-\frac{1}{2} \sum_{y \in C_{n}} p_{n, y} \ln \left(2 n \eta_{2 n-1}(y)\right)=-\ln (2 n)-\frac{1}{2} \sum_{y \in C_{n}} p_{n, y} \ln p_{n, y}=-\ln (2 n)+\frac{1}{2} H(\mathbf{p}),
$$

where $H(\mathbf{p})$ is the entropy of the probability vector $\mathbf{p}$, always bounded by $\ln \left(\operatorname{card}\left(C_{n}\right)\right)$. We thus have on the set $A_{n}$,

$$
\left|I_{n}^{1}(x)\right| \leqslant c \exp \left\{-\ln (2 n)+\frac{1}{2} \ln \left(2 d_{n, 1}\right)\right\} \prod_{y \in C_{n}}\left(\frac{1\left\{f\left(T^{y} x\right)\left(1-f\left(T^{y} x\right)\right)>0\right\}}{\sqrt{f\left(T^{y} x\right)\left(1-f\left(T^{y} x\right)\right)}}\right)^{p_{n, y}} .
$$

Applying Hölder's inequality and using the fact that $T$ preserves the measure $\mu$, we get

$$
\begin{aligned}
& \int_{E}\left|I_{n}^{1}(x)\right| d \mu(x) \leqslant C n^{-3 / 4+\delta_{1} / 2} \int_{E} \prod_{y \in C_{n}}\left(\frac{1\left\{f\left(T^{y} x\right)\left(1-f\left(T^{y} x\right)\right)>0\right\}}{\sqrt{f\left(T^{y} x\right)\left(1-f\left(T^{y} x\right)\right)}}\right)^{p_{n, y}} d \mu(x) \\
& \leqslant C n^{-3 / 4+\delta_{1} / 2} \prod_{y \in C_{n}}\left[\int_{E}\left(\frac{1\left\{f\left(T^{y} x\right)\left(1-f\left(T^{y} x\right)\right)>0\right\}}{\sqrt{f\left(T^{y} x\right)\left(1-f\left(T^{y} x\right)\right)}}\right) d \mu(x)\right]^{p_{n, y}} \\
& =C n^{-3 / 4+\delta_{1} / 2} \int_{E} \frac{1}{\sqrt{f(x)(1-f(x))}} d \mu(x) .
\end{aligned}
$$

Lemma 4 is proved.

Now, using (3.6), write with the usual notation $d_{n, 3}=n^{1 / 2+\delta_{3}}$ :

$$
\int_{E} \widetilde{\mathbf{P}}_{T}^{(x)}\left[A_{n} \backslash B_{n} \mid \mathscr{F}\right] d \mu(x) \leqslant C d_{n, 3} \int_{E}\left(\left|I_{n}^{1}(x)\right|+\left|I_{n}^{2}(x)\right|\right) d \mu(x)
$$

and consider $\delta_{3}>2 \delta_{2}$. By the previous lemmas, we have

$$
d_{n, 3} \int_{E}\left|I_{n}^{1}(x)\right| d \mu(x)=O\left(n^{-1 / 4+\delta_{3}+\delta_{1} / 2}\right), \quad d_{n, 3} \int_{E}\left|I_{n}^{2}(x)\right| d \mu(x)=O\left(e^{-n^{2 \delta_{2} / 2}}\right)
$$

and the proposition follows by choosing $\delta_{1}, \delta_{2}, \delta_{3}$ small enough. 
Combining equations (3.3), (3.4), and (3.5), we obtain (3.2) and then (3.1). By BorelCantelli's lemma, we get

$$
\tilde{\mathbf{P}}_{\mu}\left[M_{T_{n}}=(0,0) \text { i.o. }\right]=\mathbf{P}_{\mu}\left[\mathbf{P}\left[M_{T_{n}}=(0,0) \text { i.o. }\right]\right]=0,
$$

where i.o. stands for infinitely often, and thus for $\mathbf{P}_{\mu}$-almost every orientation $\epsilon, \mathbf{P}\left[M_{T_{n}}=\right.$ $(0,0)$ i.o. $]=0$. This proves the transience of $\left(M_{T_{n}}\right)_{n \in \mathbb{N}}$ for $\mathbf{P}_{\mu}$-almost every orientation $\epsilon$, and by Lemma 1 , the $\mathbf{P}_{\mu}$-almost sure transience of the simple random walk on the annealed oriented lattice. Transience in the quenched case is a direct consequence of the transience in the annealed case.

\subsection{Proof of the strong law of large numbers.}

Lemma 5 (SLLN for the embedded random walk). We have

$$
\lim _{n \rightarrow+\infty} \frac{M_{T_{n}}}{n}=(0,0) \quad \widetilde{\mathbf{P}}_{\mu} \text {-a.s. }
$$

$\mathrm{P}$ r o o f. Since $\left(Y_{n}\right)_{n \geqslant 0}$ is a simple random walk, $Y_{n} / n$ goes to $0 \widetilde{\mathbf{P}}_{\mu}$-a.s. as $n \rightarrow \infty$ and it is enough to prove that $\left(X_{n} / n\right)$ converges almost surely to 0 . Introduce

$$
Z_{n}=\sum_{k=0}^{n-1} \epsilon_{Y_{k}}=\sum_{y \in \mathbf{Z}} \epsilon_{y} \eta_{n-1}(y) .
$$

Under the probability measure $\widetilde{\mathbf{P}}_{\mu}$, the stationary sequence $\left(\epsilon_{Y_{k}}\right)_{k \geqslant 0}$ is ergodic [9], so from Birkhoff's theorem, as $n$ tends to infinity, $Z_{n} / n \rightarrow \mathbf{E}\left[\epsilon_{0}\right]=0$ a.s. Clearly, $X_{n}-m Z_{n}=$ $\sum_{y \in \mathbf{Z}} \epsilon_{y} \sum_{i=1}^{\eta_{n-1}(y)}\left(\xi_{i}^{(y)}-m\right)$ and for an even integer $r$

$$
\begin{aligned}
& \mathbf{E}\left[\left(X_{n}-m Z_{n}\right)^{r}\right] \\
& \quad=\sum_{y_{1} \in \mathbf{Z}, \ldots, y_{r} \in \mathbf{Z}} \mathbf{E}\left[\epsilon_{y_{1}} \cdots \epsilon_{y_{r}} \sum_{i_{1}=1}^{\eta_{n-1}\left(y_{1}\right)} \cdots \sum_{i_{r}=1}^{\eta_{n-1}\left(y_{r}\right)} \mathbf{E}\left[\left(\xi_{i_{1}}^{\left(y_{1}\right)}-m\right) \times \cdots \times\left(\xi_{i_{r}}^{\left(y_{r}\right)}-m\right) \mid \mathscr{F} \bigvee \mathscr{G}\right]\right]
\end{aligned}
$$

The $\xi_{i}^{(y)}$ 's are independent of the vertical walk and the orientations; moreover, the random variables $\xi_{i}^{(y)}-m, i \geqslant 1, y \in \mathbf{Z}$, are i.i.d. and centered, so the summands are nonzero if and only if $i_{1}=\cdots=i_{r}$ and $y_{1}=\cdots=y_{r}$. Then

$$
\mathbf{E}\left[\left(X_{n}-m Z_{n}\right)^{r}\right]=n \mathbf{E}\left[\left(\xi_{1}^{(0)}-m\right)^{r}\right]=: n m_{r} \quad \text { (say). }
$$

Let $\delta>0$. By Chebyshev's inequality,

$$
\mathbf{P}\left[\left|\frac{X_{n}-m Z_{n}}{n}\right| \geqslant \delta\right] \leqslant \frac{1}{\delta^{r} n^{r}} \mathbf{E}\left[\left(X_{n}-m Z_{n}\right)^{r}\right]=\frac{m_{r}}{\delta^{r} n^{r-1}} .
$$

We choose $r=4$ and thus from the Borel-Cantelli Lemma we deduce that $X_{n}-m Z_{n} / n$ converges almost surely to 0 as $n$ goes to infinity. Lemma 5 is proved.

Using similar techniques, one also proves the following result.

Lemma 6. The sequence $\left(T_{n} / n\right)_{n \geqslant 1}$ converges $\widetilde{\mathbf{P}}_{\mu}$-a.s. to $1+m$ as $n \rightarrow+\infty$.

Let us prove now the almost sure convergence of the sequence $\left(M_{n} / n\right)_{n \geqslant 1}$ to $(0,0)$. Since the sequence $\left(T_{n}\right)_{n \geqslant 1}$ is strictly increasing, there exists a nondecreasing sequence of integers sequence $\left(U_{n}\right)_{n \geqslant 1}$ such that $T_{U_{n}} \leqslant n<T_{U_{n}+1}$. Denote $M_{n}=\left(M_{n}^{(1)}, M_{n}^{(2)}\right)$, then we have $M_{n}^{(1)} \in\left[\min \left(M_{T_{U_{n}}}^{(1)}, M_{T_{U_{n}+1}}^{(1)}\right), \max \left(M_{T_{U_{n}}}^{(1)}, M_{T_{U_{n}+1}}^{(1)}\right)\right]$ and $M_{n}^{(2)}=M_{T_{U_{n}}}^{(2)}$, by definition of the embedding. The (sub-)sequence $\left(U_{n}\right)_{n \geqslant 1}$ is nondecreasing and $\lim _{n \rightarrow+\infty} U_{n}=+\infty$; then, by combining Lemmas 5 and 6 , we get that as $n \rightarrow+\infty$

$$
\frac{M_{T_{U_{n}}}}{T_{U_{n}}} \rightarrow(0,0) \quad \widetilde{\mathbf{P}}_{\mu} \text {-a.s. }
$$

Now,

$$
\left|\frac{M_{n}^{(1)}}{n}\right| \leqslant \max \left(\left|\frac{M_{T_{U_{n}}}^{(1)}}{n}\right|,\left|\frac{M_{T_{n}+1}^{(1)}}{n}\right|\right) \leqslant \max \left(\left|\frac{M_{T_{U_{n}}}^{(1)}}{T_{U_{n}}}\right|,\left|\frac{M_{T_{U_{n}+1}}^{(1)}}{T_{U_{n}}}\right|\right)
$$


and

$$
\left|\frac{M_{n}^{(2)}}{n}\right|=\left|\frac{M_{T_{U_{n}}}^{(2)}}{T_{U_{n}}}\right| \frac{T_{U_{n}}}{n} \leqslant\left|\frac{M_{T_{U_{n}}}^{(2)}}{T_{U_{n}}}\right| .
$$

From (3.8) we deduce the almost sure convergence of the coordinates to 0 and then that of the sequence $\left(M_{n} / n\right)_{n \geqslant 1}$ to $(0,0)$ as $n \rightarrow \infty$.

\subsection{Proof of the functional limit theorem.}

Proposition 2. The sequence of random processes $n^{-3 / 4}\left(X_{[n t]}\right)_{t \geqslant 0}$ weakly converges in the space $\mathscr{D}\left(\left[0, \infty[, \mathbf{R})\right.\right.$ to the process $\left(m \Delta_{t}\right)_{t \geqslant 0}$.

$\mathrm{P} \mathrm{r}$ o o f. Let us first prove that the finite dimensional distributions of $n^{-3 / 4}\left(X_{[n t]}\right)_{t \geqslant 0}$ converge to those of $\left(m \Delta_{t}\right)_{t \geqslant 0}$ as $n \rightarrow \infty$. We can rewrite for every $n \in \mathbf{N}, X_{n}=$ $X_{n}^{(1)}+X_{n}^{(2)}$, where

$$
X_{n}^{(1)}=\sum_{y \in \mathbf{Z}} \epsilon_{y}\left(\sum_{i=1}^{\eta_{n-1}(y)} \xi_{i}^{(y)}-m\right), \quad X_{n}^{(2)}=m \sum_{y \in \mathbf{Z}} \epsilon_{y} \eta_{n-1}(y) .
$$

Thanks to Theorem 3 the finite dimensional distributions of $n^{-3 / 4}\left(X_{[n t]}^{(2)}\right)_{t \geqslant 0}$ converge to those of $\left(m \Delta_{t}\right)_{t \geqslant 0}$ as $n \rightarrow \infty$. To conclude we show that the sequence of random variables $n^{-3 / 4}\left(X_{n}^{(1)}\right)_{n \in \mathrm{N}}$ converges in the $L^{2}$-norm to 0 as $n \rightarrow+\infty$. We have

$$
\mathbf{E}\left[\left(X_{n}^{(1)}\right)^{2}\right]=\mathbf{E}\left[\sum_{x, y \in \mathbf{Z}} \epsilon_{x} \epsilon_{y} \sum_{i=1}^{\eta_{n-1}(x)} \sum_{j=1}^{\eta_{n-1}(y)} \mathbf{E}\left[\left(\xi_{i}^{(x)}-m\right)\left(\xi_{j}^{(y)}-m\right) \mid \mathscr{F} \bigvee \mathscr{G}\right]\right]
$$

From the equality $\mathbf{E}\left[\left(\xi_{i}^{(x)}-m\right)\left(\xi_{j}^{(y)}-m\right) \mid \mathscr{F} \vee \mathscr{G}\right]=m^{2} \delta_{i, j} \delta_{x, y}$ we obtain

$$
n^{-3 / 2} \mathbf{E}\left[\left(X_{n}^{(1)}\right)^{2}\right]=m^{2} n^{-3 / 2} \sum_{x \in \mathbf{Z}} \eta_{n-1}(x)=m^{2} n^{-1 / 2}=o(1) .
$$

Proposition 2 is proved.

Recall that $M_{T_{n}}=\left(X_{n}, Y_{n}\right)$ for every $n \geqslant 1$. The sequence of random processes $n^{-3 / 4}\left(Y_{[n t]}\right)_{t \geqslant 0}$ weakly converges in $\mathscr{D}\left(\left[0, \infty[, \mathbf{R})\right.\right.$ to 0 , thus the sequence of $\mathbf{R}^{2}$-valued random processes $n^{-3 / 4}\left(M_{T_{[n t]}}\right)_{t \geqslant 0}$ weakly converges in $\mathscr{D}\left(\left[0, \infty\left[, \mathbf{R}^{2}\right)\right.\right.$ to the process $\left(m \Delta_{t}, 0\right)_{t \geqslant 0}$. Theorem 4 follows from this remark and Lemma 6 .

4. Examples. The main motivation of this work is the generalization of the transience results stated for the i.i.d. case in [2] to dependent or inhomogeneous orientations. We obtain various extensions corresponding to well-known examples of dynamical systems such that Bernoulli and Markov shifts, SRB measures, rotations on the torus, etc., our framework is very general from this point of view. To get the transience of the walk, we need to generate the orientations by choosing a suitable function $f$ satisfying (2.3), which requires in some sense the model not to be too close to the deterministic case: because to satisfy it, $f$ should not be « $\mu$-too often» 0 or 1 . We describe now the examples providing extensions of the i.i.d. case to various disordered orientations.

1. S h i f t s. Bernoulli and Markov shifts provide the more natural field of application of Theorem 1, including a dynamical construction of the i.i.d. case of [2] and a straightforward extension to inhomogeneous or dependent orientations. Consider the shift transformation $T$ on the product space $E=[0,1]^{\mathbf{Z}}$ endowed with the Borel $\sigma$-algebra, defined by

$$
\begin{aligned}
T: E & \longrightarrow E \\
x=\left(x_{y}\right)_{y} \in \mathbf{Z} & \longmapsto(T x)_{y}=x_{y+1} \quad \forall y \in \mathbf{Z} .
\end{aligned}
$$

Bernoulli shifts are considered when one starts from the product Lebesgue measure $\mu=$ $\lambda^{\otimes \mathbf{Z}}$ of the Lebesgue measure $\lambda$ on $[0,1]$. It is $T$-invariant and we choose as generating function $f$ the projection on the zero coordinate:

$$
\begin{aligned}
f: E & \longrightarrow[0,1], \\
x & \longmapsto x_{0} .
\end{aligned}
$$


For all $y \in \mathbf{Z}$, we then have $f \circ T^{y}(x)=x_{y}:=\xi(y) \in[0,1]$. We consider these $\xi$ 's as new random variables on $E$ whose independence is inherited from the product structure of $\mu$. The sufficient condition (2.3) becomes

$$
\int_{0}^{1} \frac{d \lambda(x)}{\sqrt{x(1-x)}}<\infty
$$

and the transience holds in this particular case. In the annealed case, the product form of $\mu$ allows another description of the i.i.d. case of [2], for which we have $\xi(y) \equiv \frac{1}{2}$ for all $y \in \mathbf{Z}$ and $\operatorname{Cov}_{\mu}\left[\epsilon_{0}, \epsilon_{y}\right]=\mathbf{E}_{\mu}\left[\epsilon_{0} \epsilon_{y}\right]=4 \mathbf{E}[\xi(0) \xi(y)]-1=0$. The result is also valid in the quenched case, for which the distribution of the orientation has an inhomogeneous product form.

If one considers a measure $\mu$ with correlations, then the same holds for $\mathbf{P}_{\mu}$. Consider, e.g., $\mu$ to be a (shift-invariant) Markovian measure on $[0,1]^{\mathbf{Z}}$ whose correlations are inherited from the shift via (2.2), with a stationary distribution $\pi$. The transience of the simple random walk on this particular dynamically oriented lattice holds then for $\mathbf{P}_{\mu}$-a.e. environment as soon as

$$
\int_{0}^{1} \frac{d \pi(x)}{\sqrt{x(1-x)}}<\infty .
$$

It is the case when the invariant measure $\mu$ is the usual Lebesgue measure or Lebesgue measure of index $p$. In the quenched case, there are no correlations by construction and the law of the orientations depends on the measurable transformation only. This case is nevertheless different from this of the Bernoulli shift, because the typical set of points $x$ for which the transience holds depends on the measure $\mu$.

2. S R B m e a s u r e s. They provide another source of examples for dependent orientations generated by transformations on the interval $E=[0,1]$. A measure $\mu$ of the dynamical system $S$ is said to be an $S R B$ measure if the empirical measure $n^{-1} \sum_{i=1}^{n} \delta_{T^{i}}(x)$ converges weakly to $\mu$ for Lebesgue almost every $x$. There exist many other definitions of SRB measures, see, e.g., [8]. In particular, it has the Bowen boundedness property in the sense that it is close to a Gibbs measure on some increasing cylinder, i.e., there exists a constant $C>0$ such that for all $x \in[0,1]$ and every $n \geqslant 1$

$$
\frac{1}{C} \leqslant \frac{\mu\left(I_{i_{1}, \ldots, i_{n}}(x)\right)}{\exp \left\{\sum_{k=0}^{n-1} \Phi\left(T^{k}(x)\right)\right\}} \leqslant C
$$

where $\Phi=-\ln \left|T^{\prime}\right|$ and $I_{i_{1}, \ldots, i_{n}}$ is the interval of monotonicity for $T^{n}$ which contains $x$.

In some cases, it is possible to control the correlations for SRB measures and we detail now an example, where our transience result holds, the Manneville-Pomeau maps introduced in the 1980's to study intermittency phenomenon in the study of turbulence in chaotic systems [1]. They are expanding interval maps on $E=[0,1]$ and the original Manneville-Pomeau map is given by

$$
\begin{aligned}
T:[0,1] & \longrightarrow[0,1], \\
x & \longmapsto T(x)=x+x^{1+\alpha} \bmod 1 .
\end{aligned}
$$

The existence of an absolutely continuous (with respect to the Lebesgue measure on $[0,1])$ SRB invariant measure $\mu$ has been established in [15] and the following bounds of Radon-Nikodým derivative $h=\frac{d \mu}{d \lambda}$ has been proved in [14]:

$$
\text { there exist } \quad C_{\star}, C^{\star}>0 \quad \text { such that } \frac{C_{\star}}{x^{\alpha}}<h(x)<\frac{C^{\star}}{x^{\alpha}} .
$$

This measure is known to be mixing, and a polynomial decay of correlation, with a power $\beta>0$, has even been proved for $g$ regular enough [7], [11], [14], [16]:

$$
\left|C_{\mu}^{g}(y)\right|=O\left(|y|^{-\beta}\right)
$$

The map $T$ is not invertible but we use the remark following Theorem 1 . It remains to find suitable function $f$ who generates orientations for which the simple random walk is 
transient. By (4.1), a sufficient condition for the condition (2.3) to hold is

$$
\int_{0}^{1} \frac{d x}{x^{\alpha} \sqrt{f(x)(1-f(x))}}<\infty
$$

and this is, e.g., true, for the function $f(x)=\frac{1}{2}(1+x-T(x))$ and the choice of an $\alpha<\frac{1}{3}$.

3. R o t a $\mathrm{t}$ i o $\mathrm{n} \mathrm{s}$. We consider the dynamical system $S=\left([0,1], \mathscr{B}([0,1]), \lambda, T_{\alpha}\right)$, where $T_{\alpha}$ is the rotation on the torus $[0,1]$ with angle $\alpha \in \mathbf{R}$ defined by

$$
x \longmapsto x+\alpha \bmod 1
$$

and $\lambda$ is the Lebesgue measure on $[0,1]$. For every function $f:[0,1] \mapsto[0,1]$ such that $\int_{0}^{1} f(x) d x=\frac{1}{2}$ and

$$
\int_{0}^{1} \frac{d x}{\sqrt{f(x)(1-f(x))}}<\infty,
$$

conclusions of Theorem 1 hold uniformly in $\alpha$. Such functions are called admissible. Every function uniformly bounded from 0 and 1 , with integral $\frac{1}{2}$ is admissible. We also allow functions $f$ to take values 0 and 1: for instance, $f_{1}(x)=x$ is admissible although $f_{2}(x)=\cos ^{2}(2 \pi x)$ is not. We actually have no explanations about this phenomenon, moreover we do not know the behavior (recurrence or transience) of the simple random walk on the dynamically oriented lattice generated by $f_{2}$.

When the generation function $f$ does not satisfy condition (2.3), a variety of results can arise by tuning the angle $\alpha$ to get different types of dynamical systems. Consider $f_{3}=1\left(\left[0, \frac{1}{2}[)\right.\right.$ and take $\alpha=1 /(2 q)$ for $q$ an integer larger than or equal to 1 ; the lattice we obtain is $\mathbf{Z}^{2}$ with undirected vertical lines and horizontal strips of height $q$, alternatively oriented to the left then to the right. The simple random walk on this deterministic and periodic lattice is known to be recurrent [2] and this provides an example of a nonergodic system, where (2.3) is not fulfilled and the walk is recurrent. When the period becomes infinite, i.e., for $\alpha=0$, the rotation is just the identity and the corresponding lattice is $\mathbf{Z}^{2}$ with undirected vertical lines and horizontal lines all oriented to the right (respectively, all to the left) when $x \in\left[0, \frac{1}{2}\left[\right.\right.$ (respectively, $x \notin\left[0, \frac{1}{2}[\right.$ ). The simple random walk on this lattice is known to be transient and this gives an example of a nonergodic system, where (2.3) is not fulfilled and the walk is transient. In the ergodic case, i.e., when $\alpha$ is irrational, we suspect the behavior of the walk to exhibit a transition according to the type of the irrational $\alpha$. When its approximation by rational numbers via a development in continuous fraction is considered to be good, i.e., when its type is large, the lattice is «quasi» periodic and the walk is believed to be recurrent. On the other hand, the walk is believed to be transient when this approximation is bad (when the type of the irrational is close to 1). A deeper study of this particular choice of dynamical system, in progress, is needed to describe more precisely the transition between recurrence and transience in terms of the type of the irrational $\alpha$.

5. Comments. We have extended the results of [2] to nonindependent or inhomogeneous orientations. In particular, we have proved that the simple random walk is still transient for a large class of models. As the walk can be recurrent for deterministic orientations, it would be interesting to perturb deterministic cases in order to get a full picture of the transience versus recurrence properties and a more systematic study of this problem is in progress. We believe that the functional limit theorem could be extended, at least to other ergodic dynamical systems, but this requires new results on random walks in ergodic random sceneries. In the i.i.d. case, M. Campanino and D. Pétritis [2] have also proved an improvement of the strong law of large numbers for the random walk in the random scenery $Z_{n}$ : almost surely, $Z_{n} / n^{\beta} \rightarrow 0$ for all $\beta>\frac{3}{4}$. Together with our functional limit theorem and the standard results for the vertical walk, this suggests the conjecture of a local limit theorem, giving a full picture of «purely random cases», for which the condition on the generation $f$ holds. This work is in progress and we also investigate the limit theorems in more general cases. 


\section{СПИСОК ЛИТЕРАТУРЫ}

1. Берже П., Помо И., Видаль К. Порядок в хаосе. М.: Мир, 1991, 368 с.

2. Campanino M., Pétritis $D$. Random walks on randomly oriented lattices. - Markov Process. Related Fields, 2003, v. 9, № 3, p. 391-412.

3. Campanino M., Pétritis $D$. On the physical relevance of random walks: an example of random walks on randomly oriented lattice. - Random Walks and Geometry. Ed. by V. Kaimanovitch. Berlin: de Gruyter, 2004, p. 393-411.

4. R. Fernández, Fröhlich J., Sokal A.D. Random Walks, Critical Phenomena, and Triviality in Quantum Field Theory. Berlin: Springer-Verlag, 1992, 444 p.

5. Guillotin N. Asymptotics of a dynamic random walk in a random scenery. I. Law of large numbers. - Ann. Inst. H. Poincaré, 2000, v. 36, № 2, p. 127-151.

6. Guillotin N. Asymptotics of a dynamic random walk in a random scenery. II. A functional limit theorem. - Markov Process. Related Fields, 1999, v. 5, № 2, p. 201-218.

7. $\mathrm{Hu} H$. Decay of correlations for piecewise smooth maps with indifferent fixed points. Ergodic Theory Dynam. Systems, 2004, v. 24, № 2, p. 495-524.

8. Jiang $M$. Sinai-Ruelle-Bowen measures for lattice dynamical systems. - J. Statist. Phys., 2003, v. 111, № 3-4, p. 863-902.

9. Kakutani S. Random ergodic theorems and Markoff processes with a stable distribution. - Proceedings of the Second Berkeley Symposium on Mathematical Statistics and Probability, 1950. Berkeley-Los Angeles: Univ. of California Press, 1951, p. 247261.

10. Keller G. Equilibrium States in Ergodic Theory. Cambridge: Cambridge Univ., 1998, 178 p. (London Math. Soc. Stud. Texts, v. 42.)

11. Liverani C., Saussol B., Vaienti S. Conformal measure and decay of correlation for covering weighted systems. - Ergodic Theory Dynam. Systems, 1998, v. 18, №6, p. $1399-1420$.

12. Kesten H., Spitzer F. A limit theorem related to a new class of self similar processes. Wahrscheinlichkeitstheor. Verw. Geb., 1979, v. 50, № 1, p. 5-25.

13. Leroux $P$. Coassociative grammar, periodic orbits, and quantum random walk over $\mathbf{Z}^{d}$. - Int. J. Math. Math. Sci., 2005. № 24, p. 3979-3996.

14. Maes C., Redig F., Takens F., van Moffaert A., Verbitski E. Intermittency and weakly Gibbs states. - Nonlinearity, 2000, v. 13, № 5, p. 1681-1698.

15. Pianigiani G. First return map and invariant measures. - Israel J. Math., 1980, v. 35, № 1-2, p. 32-48.

16. Young L.S. Recurrence times and rates of mixing. - Israel J. Math., 1999, v. 110, p. 153-188.

Поступила в редакцию

18.IX.2004

Исправленный вариант

5.I. 2006 